\title{
Comparative Assessment of Crystallographic Phase Stability of Anatase and Rutile TiO2 at Dynamic Shock Wave Loaded Conditions
}

\section{A Sivakumar}

Sacred Heart College

K Kalaiarasi

Sacred Heart College

S.Sahaya Jude Dhas

Kings Engineering College

P. Sivaprakash

Bharathidasan University

\section{S. Arumugam}

Bharathidasan University

M. Jose

Sacred Heart College

Martin Britto Dhas ( $\nabla$ martinbritto@shctpt.edu )

Sacred Heart College https://orcid.org/0000-0003-0896-7534

\section{Research Article}

Keywords: Shock waves, phase transition, TiO2 NPs, aerospace applications

Posted Date: September 27th, 2021

DOl: https://doi.org/10.21203/rs.3.rs-931997/v1

License: (9) (i) This work is licensed under a Creative Commons Attribution 4.0 International License. Read Full License

Version of Record: A version of this preprint was published at Journal of Inorganic and Organometallic Polymers and Materials on December 3rd, 2021. See the published version at https://doi.org/10.1007/s10904-021-02161-4. 


\section{Abstract}

In the present research article, authors have experimentally evaluated the shock wave resistant properties of technologically potential materials of the anatase and the rutile phase $\mathrm{TiO}_{2}$ nanoparticles at the dynamic shock wave loaded conditions. The shock wave resistant behavior has been quantitatively drawn utilizing the crystallographic phase stability of the test samples for which the required crystallographic information has been extracted from the powder XRD patterns. Based on our observed experimental results as well as the respective interpretations, it is strongly authenticated that Rutile $\mathrm{TiO}_{2}$ NPs are suitable candidates for aerospace and defense industrial applications of materials fabrications because of the outstanding shock resistant properties than that of Anatase $\mathrm{TiO}_{2} \mathrm{NPs}$ which undergo the crystallographic phase transition of rutile- $\mathrm{TiO}_{2}$ at shocked conditions.

\section{Introduction}

Shock wave experiments on materials could throw light anew each time when a systematic analysis is undertaken such that they have the prospective potential of rekindling of innovation for the state-of-theart modern technology which maneuvers the researchers to take up the task of unearthing the hidden wonders of functional materials as there are a lot of possible scope for aerospace and defense applications. Simulation of space environmental effects on materials as that of the effect of electromagnetic radiation, high energy rays, (gamma, cosmic, electron and proton) high temperature, shock waves and vibration conditions are getting remarkable attention among the fraternity of researchers due to the importance of understanding the structural, electrical, mechanical and optical stability of materials to be used in space environments. Moreover, these kinds of studies are very much essential so as to simplify the complexity of the material's selection in such a way that materials which can withstand extreme conditions could attract the possible and viable applications and also, they would help for the design, development of novel materials, improving safety, reducing the design cycle time as well as introducing various structural theory concepts for the future space applications [1-4]. The advantageous and superior high frequency applications are still the mostly sought-after to a great extend only by theoretical models whereas experimental models come up with a few shortcomings due to the lack of suitable detectors and high-power laser operating devices such that the practical applications are stymied by these factors. Hence, a lot of research work continues to progress with great vigor so as to identify high stability materials which are steady at extreme conditions and conducive for the required applications. Amidst all types of extreme environmental conditions, the dynamic shock waves can also provide a similar space environment at the ambient condition that can be achieved even in the laboratory conditions $[5,6]$. While shock waves are lounging on a material, it simultaneously generates high density, pressure, temperature, tension and force that are the special characteristic features of shock waves and they have tremendous amount of technological importance in the materials science research. As a consequence of this, shock wave induced research on crystalline as well as non crystalline materials has gradually gained momentum in recent years [7-10]. Particularly in nano - crystalline materials of metal oxides, there have been impressive results documented by several research groups including our research 
group in the last couple of years [11-23]. So far, crystallographic phase stabilities of several technologically important metal oxide NPs and their shock resistant profiles have been reported and they are $\mathrm{ZrO}_{2}(\mathrm{Fm}-3 \mathrm{~m})$ [11], $\mathrm{ZnO}\left(\mathrm{Pb}_{3} \mathrm{mc}\right)$ [12] a-MnO 2 (I4/m) [13], $\mathrm{TiO}_{2} \mathrm{NPs}\left(14{ }_{1} / \mathrm{amd}\right)$ [14-17], $\mathrm{Co}_{3} \mathrm{O}_{4}(\mathrm{Fd}-3 \mathrm{~m})$ [18], $\mathrm{NiO}(\mathrm{Fm}-3 \mathrm{~m})$ [19], $\mathrm{CeO}_{2}$ (Fm-3m) [20] $\mathrm{CuO}$ (Bmm2) [21] a- $\mathrm{Fe}_{2} \mathrm{O}_{3}$ (R-3c) [22], $\mathrm{AgO}$ (P2 $1 / \mathrm{c}$ ) [23]. Among the listed NPs, Anatase $\mathrm{TiO}_{2}, \mathrm{ZrO}_{2}$ and $\mathrm{Co}_{3} \mathrm{O}_{4}$ undergo the crystallographic phase transitions at shocked conditions $[11,16,20]$. a- $\mathrm{Fe}_{2} \mathrm{O}_{3}$ NPs undergo significant reduction in crystallinity [15]. But, the rest of materials exhibit the stable crystal structure at shocked conditions. It is well known that the crystal structure leads to the actual function of the properties of materials and hence the understanding about the crystal structure and its crystallographic phase stability against the external stimuli such as temperature and pressure is highly valid before suggesting them for any commercial applications. According to the literature, $\mathrm{TiO}_{2}$ crystallizes in several crystallographic phases such as anatase $\left(14_{1} / \mathrm{amd}\right)$, rutile $\left(\mathrm{P}_{2} / \mathrm{mnm}\right)$, brookite ( $\left.\mathrm{Pbca}\right), \mathrm{TiO}_{2}-\mathrm{B}\left(\mathrm{C}_{2} / \mathrm{m}\right), \mathrm{TiO}_{2}-\mathrm{R}(\mathrm{Pbnm}), \mathrm{TiO}_{2}-\mathrm{H}(14 / \mathrm{m})$, pyrite (Pa-3), columbite $(\mathrm{Pbcn})$ and baddelyite $\left(\mathrm{P} 2_{1} / \mathrm{c}\right)$, cotunnite, a-PbO${ }_{2}$ type $\mathrm{TiO}_{2}$, fluorite ( $\left.\mathrm{Fm} 3-\mathrm{m}\right)$, cubic and post contunnite phases [24-26]. Based on the analysis on the above listed crystal structures, the primary phases of anatase, rutile and brookite phases of $\mathrm{TiO}_{2} \mathrm{NPs}$ are being widely used in industrial applications [27]. Among these three crystal structures, the high impact of shock waves on anatase phase has received much attention such that several research groups including our group have found the crystallographic phase transitions from anatase to rutile at shocked conditions [11-14]. But, to date, no report has been found on the rutile phase $\mathrm{TiO}_{2}$ at shocked conditions. Rutile- $\mathrm{TiO}_{2} \mathrm{NPs}$ are the right candidates for electronic device applications due to the high dielectric constant and absorption of ultraviolet radiation [27]. Hence, assessment on the stability of crystal structure for the rutile phase is very much essential as that of the anatase phase at shocked conditions. In this context, we intend to address the outcome of the phase stability of the rutile- $\mathrm{TiO}_{2}$ at shocked conditions.

In this research work, a systematic investigation has been carried out on the crystallographic phase stability of the anatase and the rutile $-\mathrm{TiO}_{2} \mathrm{NPs}$ at dynamic shock wave loaded conditions and the observed results are compared between the two so as to understand better the phase stability of the anatase as well as the rutile $-\mathrm{TiO}_{2}$.

\section{Experimental Sections}

In order to obtain the anatase and rutile $\mathrm{TiO}_{2} \mathrm{NPs}$, the typical literature procedure was pursued [14] and the clear details have been provided in the supplementary section. After synthesizing the anatase and rutile $\mathrm{TiO}_{2}$, dynamic shock waves have been loaded on the samples for which the required shock waves have been generated by a shock tube (Semi-automated Reddy tube). The details of the working of the shock tube and generation of shock waves have been discussed in our earlier publications $[18,29]$ and details of the shock wave loaded procedure have been presented in the supplementary section. In the present case, shock waves of Mach number 2.2 that has the transient pressure and temperature of 2.0 $\mathrm{MPa}$ and $864 \mathrm{~K}$, respectively are utilized. Totally, four samples have been prepared for each phase (the 
anatase and the rutile). One sample each has been kept as the control sample while the respective remaining three samples for each phase have been sent for shock wave loading experiments. 30, 60 and 90 shock pulses have been loaded on the samples.

\section{Results And Discussion}

\section{XRD}

Powder X-ray diffractometric analysis has been performed to assess the crystallographic phase stabilities of the title materials. As seen in Fig. 1a, it is noticed that the control test sample has the anatase phase which is very much evident from the high intensity characteristic peak (101) at the diffraction angle $25^{\circ}$ [14]. In addition to that, it has some minor diffraction peaks at $41.26^{\circ}, 62.63^{\circ}, 64.68^{\circ}$ which belong to the rutile phase. While considering the rutile phase, the corresponding diffraction planes are (110), (101), (200), (111), (210), (211), (220), (002), (310), (301), and (112). The observed XRD pattern is well-matched with ICDS data base code number 9161 and the corresponding XRD pattern is presented in Fig. 1b. At shock wave loaded conditions, there is no significant change observed up to 60 shocks in terms of peak shift and peak broadening of (101) whereas at 90 shocks, the mother phase of the test sample is turned into the rutile phase by the impact of shock waves and the observed phase sequence with respect to number of shock waves is well consistent with our previous observation [14]. There are number of valid reasons behind the transition of the anatase to rutile phase at shocked conditions that are prerequisites so as to understand better the shock wave induced phase transition. As the possible scientific reasons behind the particular phase transition are analyzed, it is found in the literature that the crystallographic parameters of the anatase phase are $a=3.785, b=3.785$, and $c=9.514 \AA$ and it has the tetragonal crystal structure [14]. The anatase phase has significantly longer c-axis than that of both a and $\mathrm{b}$ axes so that c-axis has more compressibility than the other two axes and it can be considered as a prominent key point for the occurrence of the phase transition. On the other hand, the anatase phase has octahedral population which has hard occupied $\left(\mathrm{TiO}_{6}\right)$ and soft empty $\left(\mathrm{O}_{6}\right)$ sites $[30,31]$.

Hence, there are plenty of possibilities for the anatase phase to be changed into another phase which is controlled by the external parameters such as pressure and temperature. During the shock wave loaded conditions, the c-axis may break along its length in-local coordination bond network especially the bonds between $\mathrm{Ti}-\mathrm{O}(1)$ and $\mathrm{Ti}-\mathrm{O}(2)$ of anatase which in turn trigger the formation of next possible phase of the mother compound. Moreover, during the bond breakage of the anatase $\mathrm{TiO}_{2} \mathrm{NPs}$ that are formed along with the c-axis could suffer significant volume reduction or compression caused by the impact of shock waves. Hence at this stage, the rutile phase has to be the next possibility to occur due to the impact of shock waves. Since rutile belongs to the tetragonal phase $\left(\mathrm{P}_{2} / \mathrm{mnm}\right)$, the geometrical values are $\mathrm{a}=$ $4.593, b=4.593$ and $c=2.959 \AA[30,31]$. During the shock wave loaded condition, the anatase phase has experienced significant reduction of length in c-axis whereas slight enhancement is observed for $a$ and baxes so that the rutile phase has emerged at the shocked conditions. Moreover, the required time is highly dependent on the values of transient pressure and temperature with which the anatase to the rutile phase 
transition has been achieved at 90 shocks. But in the case of the rutile phase, following with the same number of shocks and the same interval i.e., from 0 to 90 shocks, neither peak shift nor phase change have been observed. The zoomed versions of the XRD patterns are presented in Fig. 2 for better visibility. Based on the observed results, it is quite clear that, the anatase phase is less stable than that of the rutile phase which is well consistent with the reported thermodynamic stability profiles [27].

While considering the crystal structure of rutile, it belongs to the tetragonal unit cell having titanium cations and oxygen anions. The coordination numbers of the titanium cations and oxygens are six and three, respectively. It could be noted that the local structure of $\mathrm{TiO}_{6}$ octahedron which give rise to the basic building unit has the resemblance as in the rutile and anatase, whereas the connection existing between the neighboring $\mathrm{TiO}_{6}$ octahedra is not the same for both the phases. To be more specific, every $\mathrm{TiO}_{6}$ octahedron found in anatase is connected to 8 neighboring octahedra (four by edge sharing and four by corner-sharing) while each $\mathrm{TiO}_{6}$ octahedron in rutile has the connection of 10 neighboring octahedra (two by edge sharing, and eight by corner-sharing) [27]. The sharing edges of the $\mathrm{TiO}_{2}$ may lead to the high shock resistance of the rutile $-\mathrm{TiO}_{2}$ at shocked conditions. As seen in Fig. $2 \mathrm{~b}$, the characteristic diffraction peak position of the rutile phase (110) remains the same with respect to the number of shock pulses and the shape of the diffraction peak is not changed. It is a key point to be considered for high shock resistant materials. On the other hand, while looking at the actual crystal structure of the rutile, the adjacent distance between $\mathrm{Ti}$ atoms is significantly lower as compared to the anatase. As seen in Fig. 3, the length of adjacent Ti atoms and bond angle is significantly low compared to the anatase phase. As mentioned above, the rutile phase has a more closely packed crystal structure as compared to the anatase as well as the gap between the most occupied (valence band) state and the least occupied (conduction band) state. The structural features of the rutile phase with lower $k$ value enables the interactions stronger that occur between Ti-3d and 0-2p which give rise to significantly higher band dispersion in the rutile phase than that of the anatase [32, 33]. So that, the interaction between $\mathrm{Ti}-\mathrm{O}-\mathrm{Ti}$ is much higher than that of the anatase phase that may also lead to acquire high shock resistance. In addition to that, in the case of $\mathrm{TiO}_{2}$, the values of surface energy of the anatase (101) and rutile (110) phases are found to be $0.51 \mathrm{Jm}^{-2}$ and $0.60 \mathrm{Jm}^{-2}$, respectively [32, 33]. Based on the crystallographic point of view and chemical point of view, the rutile phase $\mathrm{TiO}_{2}$ has a highly favorable crystal structure configuration than that of the anatase phase and also high shock resistant behavior so that the rutile- $\mathrm{TiO}_{2}$ can be a potential material for the applications of device fabrication. Figure 4 provides the possible mechanism of the crystal phase transitions form the anatase to the rutile phase at shocked conditions.

As seen in Fig. 4, the horizontal Ti-O bonds might have broken at 90 shocked conditions so as to form the rutile phase $\mathrm{TiO}_{2}$ that are marked in ellipsoids in Fig. 5. But there is no crystallographic and lattice deformation that is found for the rutile $\mathrm{TiO}_{2}$ at shocked conditions. Furthermore, as seen in Fig. $2 \mathrm{a}$ and Fig. $3 a$, there is considerable signature of the anatase phase in the shock wave induced rutile phase. The percentage of the presence of the anatase and the rutile phases at 90 shocked conditions is calculated using standard formulation [12]. 
During the shock wave induced phase transition of the anatase to the rutile, the anatase phase is not completely converted to the rutile phase wherein the respective ratio of the phases are $9.173 \%$ and $90.827 \%$ for the anatase and the rutile phase at 90 shocked conditions. In the case of rutile, no change is found.

\section{Conclusion}

The anatase and the rutile $\mathrm{TiO}_{2} \mathrm{NPs}$ have been synthesized by hydrothermal method and the crystallographic phase stabilities have been assessed at dynamic shock wave loaded conditions and the performances of phase stability against the impact of shock waves have been evaluated by X-ray diffraction technique. Based on the observed X-ray diffraction pattern of the anatase- $\mathrm{TiO}_{2}$, crystallographic transition is authenticated as to be the rutile- $\mathrm{TiO}_{2}$ at 90 shocked conditions. But considerable amount of the anatase phase is still present in the mother sample. In the case of the rutile phase, there is no change observed and also not even diffraction peak shift is observed. Hence, based on the obtained X-ray diffraction patterns, it could be considered that the rutile phase $\mathrm{TiO}_{2}$ has high shock resistance than that of the anatase phase promoting it to be a potential material for aerospace and defense electronic device applications.

\section{Declarations}

\section{Acknowledgment:}

The authors thank Department of Science and Technology (DST), India for funding through DST-FIST programme (SR/FST/College-2017/130 (c))

\section{Conflict of interest statement:}

The authors declare no conflicts of interest regarding this article.

\section{References}

1. Yaping Chen, Ruijing Fu, Lingrui Wang, Zhiwei Ma, Guanjun Xiao, Kai Wang, and Bo Zou, J. Mater. Chem. A, 7, 6357-6362 (2019)

2. A. V. Krasheninnikov and K. Nordlund, Appl. Phys. 107, 071301 (2010)

3. Takara Suzuki, Yuta Saito, Paul Fons, Alexander V. Kolobov, Junji Tominaga, and Muneaki Hase, Appl. Phys. Lett. 111, 112101 (2017)

4. I.Kim, Y.Yang, G. Park, S. M. Jo, Acta Astronautica, 181, 52-60 (2021).

5. A. Sivakumar and S. A. Martin Britto Dhas, J. Appl. Cryst. 52, 1016-1021 (2019)

6. Vishakantaiah Jayaram, Ravichandran Ranjith, Parthasarathi Bera, J.Mater.Sci.Appl 4,37-46 (2018)

7. P. Renganathan , and Y. M. Gupta, J. Appl. Phys. 126, 115902 (2019) 
8. A. Sivakumar, S. Sahaya Jude Dhas, T. Pazhanivel, Abdulrahman I. Almansour, Raju Suresh Kumar, Natarajan Arumugam, C. Justin Raj, and S. A. Martin Britto Dhas, Cryst. Growth Des. 21, 1617-1624 (2021)

9. Shamal L. Chinke, Inderpal Singh Sandhu, D. R. Saroha, and Prashant S. Alegaonkar, ACS Appl. Nano Mater. 1, 6027-6037 (2018)

10. Hongxiang Zong, Ping He, Xiangdong Ding, and Graeme J. Ackland, Phys.Rev. B 101, 144105 (2020) 11. V. Jayaram, K. P. J. Reddy, Adv. Mater. Lett. 7, 100-150 (2016)

12. A.Sivakumar, C.Victor, M.Muralidhr Nayak and S.A. Martin Britto Dhas, Mater. Res. Express 6, 045031 (2019)

13. A. Rita, A. Sivakumar, S. Sahaya Jude Dhas, and S. A. Martin Britto Dhas, J Mater Sci: Mater Electron 31, 20360-20367 (2020)

14. S. Kalaiarasi, A. Sivakumar, S.A. Martin Britto Dhas, M. Jose, Mater. Lett. 219 72-75 (2018)

15. V. Jayaram, Preetam Singh, K.P.J. Reddy, Adv. Ceram. Sci. Engg 2, 40-46 (2013)

16. Xiang Ga, Jianjun Liu, Pengwan Chen, Mater.Res.Bull 44, 1842-1845 (2009)

17. Jianjun Liua, Yingchun Yu, Hongliang He, Xiaogang Jin, Kang Xu, Mater.Res.Bull. 35, 377-382 (2000)

18. A. Sivakumar, S. Soundarya, S. Sahaya Jude Dhas, K. Kamala Bharathi, and S. A. Martin Britto Dhas, J.Phys.Chem.C 124, 10755-10763 (2020)

19. A. Rita, A. Sivakumar, S. A. Martin Britto Dhas, J Supercond Nov Magn 33, 1845-1849 (2020)

20. Vishakantaiah Jayaram, Asha Gupta, K.P.J.Reddy, J.Adv.Ceram. 3, 297-305 (2014)

21. A. Rita, A. Sivakumar, S. A. Martin Britto Dhas, J.Nanostrct.Chem. 9, 225-230 (2019)

22. A.Rita, A.Sivakumar, M.Jose and S.A.Martin Britto Dhas, Mater. Res. Express 6, 095035 (2019)

23. A. Rita, A. Sivakumar, S. Sahaya Jude Dhas, S. A. Martin Britto Dhas, J. Nanostruct. Chem. 10, 309$316(2020)$

24. I. Kim, H. Shim, YH. Kim, G. Park, Mater.Lett. 294, 129793 (2021)

25. Quanjun Li, Ran Liu, Bingbing Liu, Lin Wang, Kai Wang, Dongmei Li, Bo Zou, Tian Cui, Jing Liu, Zhiqiang Chenc and Ke Yang, RSC Adv 2, 9052-9057 (2012)

26. S. Kalaiarasi, S.A.Martin Britto Dhas, M.Jose and S.Jerome Das, Phase.Trans 93, 1-12 (2020)

27. Dorian A. H. Hanaor, Charles C. Sorrell, J Mater Sci 46, 855-874 (2011)

28. Yu. M. Shulga, D. V. Matyushenko, A. A. Golyshev, D. V. Shakhrai, A. M. Molodets, E. N. Kabachkov, E.N. Kurkin, and I. A. Domashnev, Tech. Phys. Lett. 36, 841-843 (2010).

29. A. Sivakumar, S. Balachandar, S. A. Martin Britto Dhas, Hum.Fact.Mech.Eng.Defen. Safety 4, 3 (2020)

30. Xudong Wang, Zhaodong Li, Jian Shi, and Yanhao Yu, Chem. Rev 114, 9346-9384 (2014)

31. Muhammad Tayyab Noma, Muhammad Azeem Ashraf, Azam Ali, Environ. Sci. Pollut. Res 26, 32623291 (2019) 
32. Matheus de Aquino Barbosaa, Guilherme da Silva Lopes Fabrisa, Mateus Meneghetti Ferrera, Douglas Henrique Marcelino de Azevedoa, Julio Ricardo Sambrano, Mater.Res. 20, 920-925 (2017)

33. Juan Andrés, Lourdes Gracia, Amanda Fernandes Gouveia, Mateus Meneghetti Ferrer and Elson Longo, Nanotechnology 26, 405703 (2015)

\section{Figures}
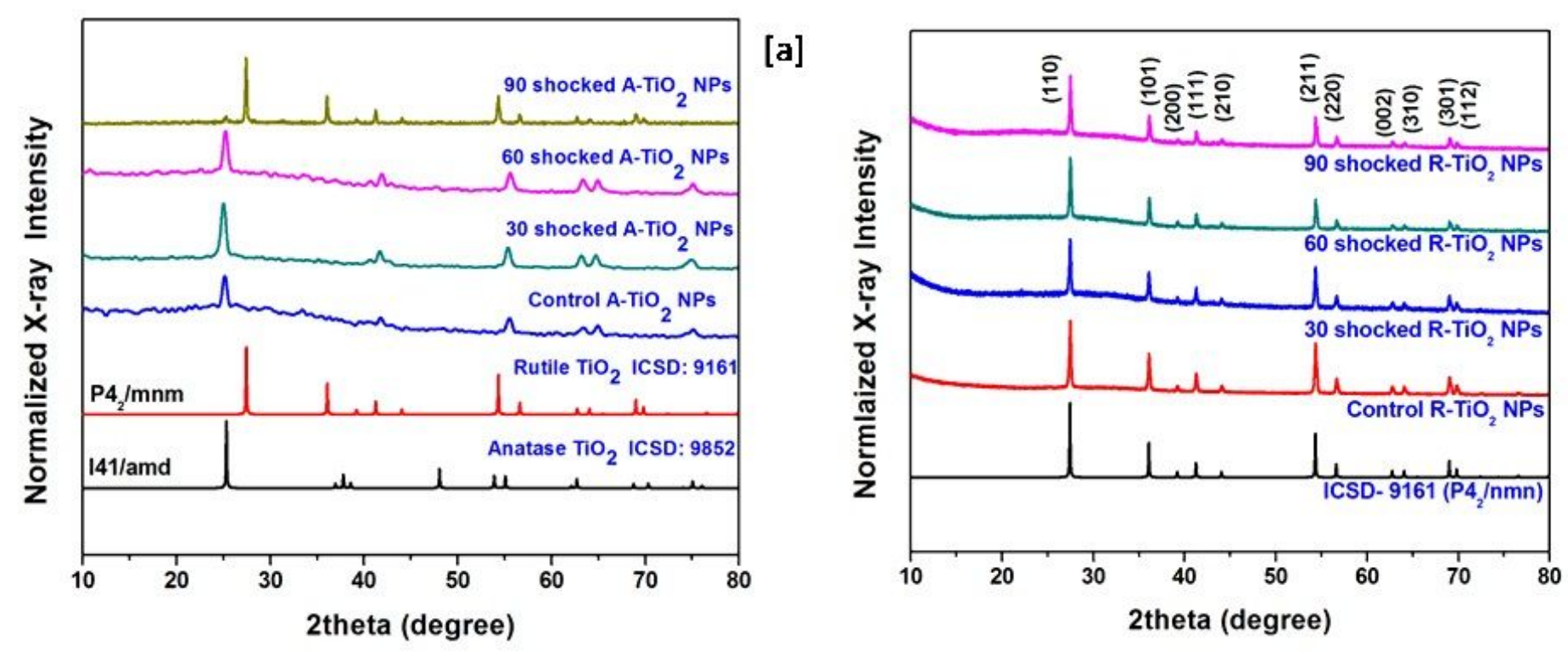

[b]

Figure 1

XRD patterns for the control and shocked samples (a) anatase TiO2 NPs (b) Rutile-TiO2 

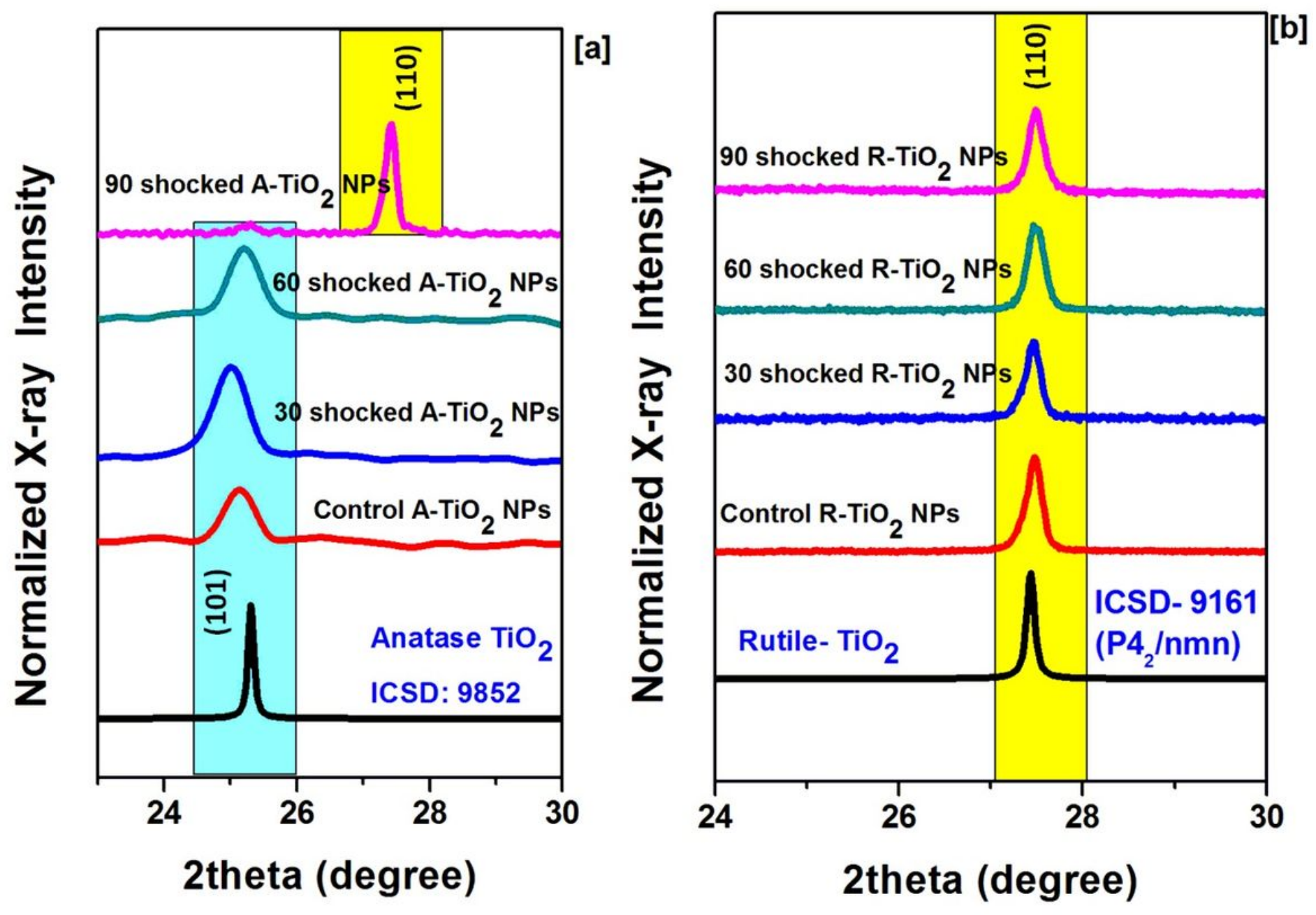

Figure 2

Characteristic peaks of XRD patterns for the control and shocked samples (a) anatase TiO2 NPs (b) Rutile-TiO2 


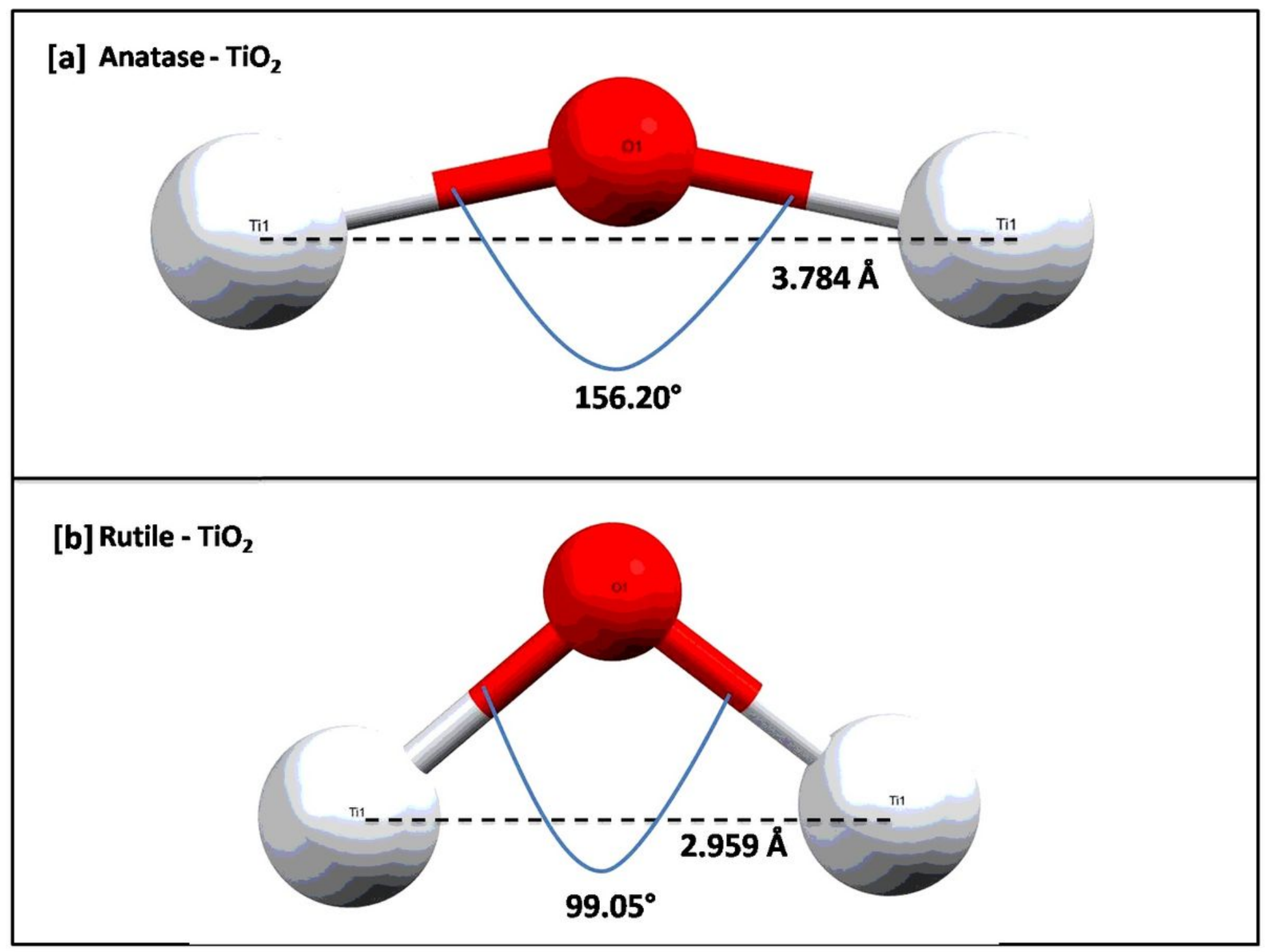

Figure 3

Adjacent bond lengths of Ti atoms (a) Anatase [CIF- ICSD 9852] (b) Rutile [CIF- ICSD -9161] 


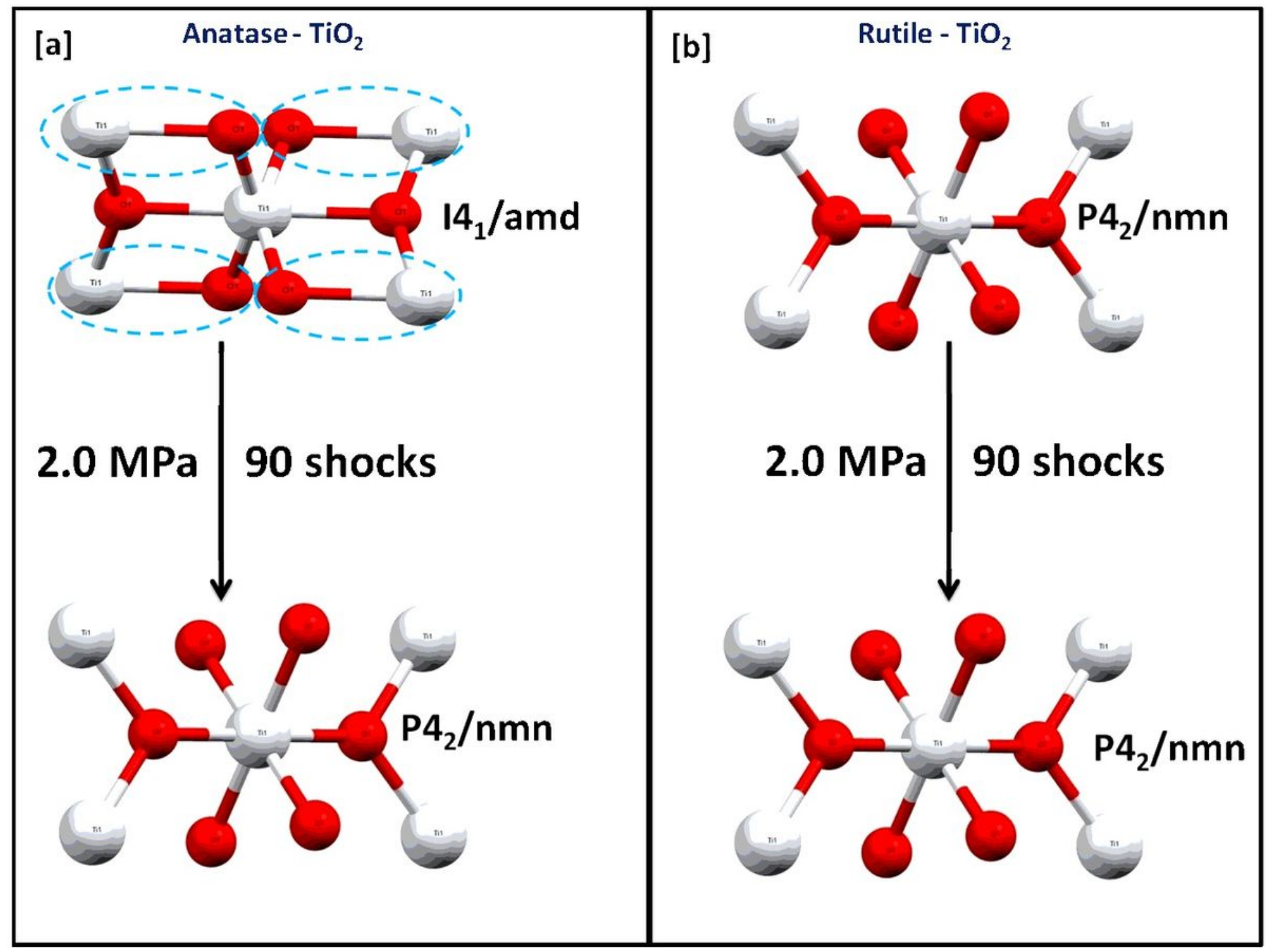

Figure 4

Schematic diagram of the possible response of the TiO2 phases at 90 shocks (a) the anatase (b) the rutile. The blue dotted line indicates the bond between $\mathrm{Ti}$ and $\mathrm{O}$ atoms which can break and induce the phase transitions.

\section{Supplementary Files}

This is a list of supplementary files associated with this preprint. Click to download.

- AnataseRutileTiO2ESIfiles.doc 